\title{
miR-145 suppresses colorectal cancer cell migration and invasion by targeting an ETS-related gene
}

\author{
SHULING LI ${ }^{1,2}$, XIAOBING WU ${ }^{1}$, YUANDONG XU ${ }^{1}$, SHANGBIAO WU $^{1}$, \\ ZHIFA LI ${ }^{1}$, RONG CHEN ${ }^{1}$, NANQI HUANG ${ }^{1}$, ZIYUAN ZHU ${ }^{1}$ and XUEHU XU ${ }^{1}$ \\ ${ }^{1}$ Department of Gastrointestinal Surgery, The Third Affiliated Hospital of Guangzhou Medical University; \\ ${ }^{2}$ Key Laboratory for Major Obstetric Diseases of Guangdong Province and Key Laboratory of Reproduction \\ and Genetics of Guangdong Higher Education Institutes, Guangzhou, Guangdong, P.R. China
}

Received January 29, 2016; Accepted March 17, 2016

DOI: $10.3892 /$ or.2016.5042

\begin{abstract}
MicroRNA-145 (miR-145) has been demonstrated to be downregulated in various cancer types including colorectal cancer (CRC). However, the function of miR-145 in CRC has not been clearly elucidated. In this study, we examined miR-145 expression by quantitative real-time PCR (qRT-PCR) in CRC cell lines as well as tumors and corresponding normal mucosa, and the results were correlated to the clinicopathological parameters. In addition, using computational algorithms we investigated putative miR-145 targets. The role of miR-145 was further examined in studies in vitro. In our study miR-145 was significantly decreased in CRC tissues and cell lines compared with non-cancerous colorectal mucosa, especially lymph node or distance metastasis cases. Based on computational algorithms, we assumed that ERG was directly modulated by miR-145 in colorectal cancer cells. For the first time, we demonstrated that ERG was highly expressed in CRC tissues compared with normal ones by qRT-PCR. The inverse correlation between the expression of miR-145 and ERG was observed in CRC tissues. Dual-Luciferase assays demonstrated the direct interaction between miR-145 and 3'-UTR of ERG mRNA. Ectopic expression of miR-145 suppressed the proliferation and invasion ability of colorectal cancer cells, while ERG knockdown partially restored the tumor suppressive effect of miR-145. These results suggested that miR-145 might act as a tumor suppressor during the process of CRC malignant transformation by interacting with ERG.
\end{abstract}

Correspondence to: Dr Xuehu Xu, Department of Gastrointestinal Surgery, The Third Affiliated Hospital of Guangzhou Medical University, 63 Duobao Road, Guangzhou, Guangdong 510150, P.R. China

E-mail: xuehu_xu@126.com

Key words: colorectal cancer, ETS-related gene, miR-145, migration, invasion

\section{Introduction}

Colorectal cancer (CRC) is the third most common cancer and the third-leading cause of cancer death in the United States (1). Although most CRC patients undergo surgical resection of tumors, regional and distant metastases strongly impact 5-year survival rates (2). Unfortunately, treatments of patients with metastatic CRC are not very efficient. Therefore, it is of great importance to further explore the underlying molecular mechanisms of CRC progression in order to develop new therapeutic strategies, especially for patients with metastatic CRC.

Erythroblast transformation-specific (ETS)-related gene (ERG) belongs to the ETS transcription factor family, and plays an important physiological role in hematopoiesis (3), angiogenesis (4), and bone development (5). In addition, aberrant ERG expression has been observed in solid tumors and leukemia (6,7). Furthermore, ERG gene fusion proteins such as transmembrane protease, serine 2 (TMPRSS2)-ERG have been associated with poor prognosis in prostate cancer (8). In prostate cancer, ectopic ERG expression may induce acquired invasive traits and endothelial mesenchymal transition (EMT) (9). Although an increasing number of studies indicate that ectopic ERG expression is involved in the pathogenesis of various solid tumors and hemopathies, further studies are still required to fully elucidate the underlying biological mechanisms of the ERG action in tumorigenesis.

microRNAs (miRNA or miR) are single strand non-coding RNAs composed of 21-24 nucleotides, which exert their function by interacting with the complementary sites in the 3' untranslated region (UTR) of target mRNAs. miRNAs regulate multiple cellular functions such as cell proliferation, apoptosis and differentiation (10). In addition, it has been shown that aberrant miRNA expression results in ectopic expression of different gene products, which may consequently lead to carcinogenesis and tumor progression (11).

miR-145 was first reported to be consistently decreased in precancerous colorectal lesions as well as in different stages of colorectal cancer tissue samples compared to normal colorectal mucosa (12). Since then, increasing evidence has shown that ERG expression is reduced in different types of cancer, supporting the idea that miR-145 acts as a tumor 
suppressor in breast cancer (13), prostate cancer (14) and neuroblastoma (15). In addition, it has been shown that ectopic expression of miR-145 influences cell cycle distribution, invasion and differentiation level of tumor cells in CRC (16). Contrary to these reports, Arndt et al showed that miR-145 may have an oncogenic role in CRC by suppressing E-cadherin expression and promoting anchorage-independent growth in vitro (17). Therefore, depending on the cell lines and cancer types, miR-145 may have either a tumor suppressive or an oncogenic effect. Hence, the exact miR-145 function and regulation in $\mathrm{CRC}$ is still unknown and demands further investigation.

Therefore, we undertook to further investigate the role of miR-145 in tumorigenesis as well as its relationship to ERG in clinical samples of colorectal tumors and CRC cell lines in vitro.

\section{Materials and methods}

Clinical specimens. In this study, 48 pairs of colorectal tumors and corresponding normal mucous tissue $(5 \mathrm{~cm}$ away from the cancer lesions) were collected from colorectal cancer patients who underwent colorectal resection at the Third Affiliated Hospital of Guangzhou Medical University. Tissue samples were snap-frozen in liquid nitrogen and then stored at $-80^{\circ} \mathrm{C}$ until further use. The pathological diagnosis of CRC specimens and confirmation of the adjacent normal intestinal mucosa were performed by at least two pathologists. The TNM classification was performed according to the National Comprehensive Cancer Network (NCCN) guideline (18). None of the patients received pre-operative chemotherapy or radiotherapy. Patients with other malignancies were excluded. The Clinical Research Ethics Committee of the Third Affiliated Hospital of Guangzhou Medical University approved the research protocols. Written informed consents were obtained from all patients.

Cell lines and treatment. Human embryonic kidney cell line HEK-293T and five human CRC cell lines (RKO, DLD-1, HCT-116, SW620 and SW480) were obtained from the American Type Culture Collection (ATCC; Rockville, MD, USA). All cell lines were cultured in Dulbecco's modified Eagle's medium (DMEM; Gibco-BRL, Grand Island, NY, USA) supplemented with $10 \%$ fetal bovine serum (FBS; HyClone, Logan, UT, USA) and $1 \%$ penicillin/streptomycin, and cultured in humidified incubator at $37^{\circ} \mathrm{C}$ and supplemented with $5 \% \mathrm{CO}_{2}$.

Total RNA isolation and quantitative real-time PCR. Total RNA from clinical specimens and CRC cell lines was isolated with TRIzol (Invitrogen Life Technologies, Carlsbad, CA, USA) according to the manufacturer's protocol. A NanoDrop 2000 spectrophotometer (Thermo Fisher Scientific, Madrid, Spain) was used to measure RNA concentration. Next, reverse transcription reactions were performed with PrimeScript RT Reagent kit (Takara, Dalian, China), and quantitative real-time PCR (qRT-PCR) was performed using SYBR Premix DimerEraser (Takara) and the Step One Plus Real-time PCR system (Applied Biosystems, Foster City, CA, USA). For miR-145 detection, miRNA-specific primers were purchased from Shanghai Generay Biotechnology Co., Ltd. (Shanghai, China), and the relative miR-145 expression level was normalized to U6 expression. ERG expression was normalized to glyceraldehyde-3-phosphate dehydrogenase (GAPDH) expression. Primer sequences were as follows: miR-145 reverse transcription, 5'-TGGTGTCGTGGAG TCG-3'; miR-145 sense, 5'-ACACTCCAGCTGGGGTCCAGT TTTCCCAGGAA-3' and antisense, 5'-CTCAACTGGTGT CGTGGAGTCGGCAATTCAGTTGAGAGGGAT-3'; ERG forward, 5'-ATCGTGCCAGCAGATCCTAC-3' and reverse, 5'-CCTTGGTCATCTTGCACAGTTC-3'; GAPDH forward, 5'-CGCTGAGTACGTCGTGGAGTC-3' and reverse, 5'-GCT GATGATCTTGAGGCTGTTGTC-3'. The $2^{-\Delta \Delta \mathrm{Ct}}$ method was used for relative quantification. All reactions were performed in triplicate.

Western blot analysis. Proteins from tissue samples and CRC cell lines were extracted with lysis buffer and protein concentration was determined by Enhanced BCA Protein Assay kit (both from Beyotime Institute of Biotechnology, Haimen, China). Protein lysate was separated on $10 \%$ SDS-PAGE gels, transferred onto a PVDF membrane (Millipore, Billerica, MA, USA), and blocked with 5\% non-fat dried milk. Next, membranes were incubated with antibodies against ERG (1:2,000) (ab133264; Abcam, Cambridge, MA, USA) or GAPDH (1:5,000) (ProteinTech, Chicago, IL, USA) overnight at $4^{\circ} \mathrm{C}$, washed with Tris-buffered saline with Tween (TBST) four times, and incubated with horse raddish peroxidase (HRP) labeled anti-rabbit secondary antibody $(1: 5,000)$ for $1 \mathrm{~h}$ at room temperature. Enhanced chemiluminescence (ECL) reagent (Pierce, Rockford, IL, USA) was used to visualize protein bands according to the manufacturer's protocol. The relative density of the western blot bands was measured by ImageJ (19) and normalized to sample T1.

Oligonucleotide transfection. For the oligonucleotide transfection experiments miR-145 mimics, miR-145 inhibitors and corresponding normal controls were purchased from RiboBio (Guangzhou, China). ERG siRNAs and non-targeting siRNA were obtained from GenePharma (Shanghai, China). siRNA sense and antisense sequences were as follows: 5'-GACGUCAACAUCUUGUUAUTT-3' and 5'-GACGUC AACAUCUUGUUAUTT-3'. Oligonucleotide transfection was performed according to the manufacturer's protocol using Lipofectamine 2000 (Invitrogen Life Technologies). In brief, $50 \mathrm{nmol} / 1$ of miR-145 mimics or $100 \mathrm{nmol} / \mathrm{l}$ of miR-145 inhibitors were used in transfections. For knockdown of endogenous ERG $100 \mathrm{nmol} / \mathrm{l}$ of ERG siRNA was used.

Luciferase reporter assay. We used Targetscan (http://www. targetscan.org) for the prediction of putative miR-145 targets. Using this analysis the ERG (NG_029732) 3'-UTR region was predicted to have putative binding site complementary to the seed region of miR-145. In order to construct the plasmid containing this site, $1.5 \mathrm{~kb}$ sequences of the ERG 3'-UTR region were amplified from human genomic DNA, and inserted into restriction sites ( $S g f \mathrm{I}$ and $N o t \mathrm{I})$ of Dual-Luciferase reporter psiCHECK 2 plasmid (Promega, Madison, WI, USA) and this plasmid was denoted as psiCHECK2-WT. The Dual-Luciferase reporter plasmid containing a mutated 

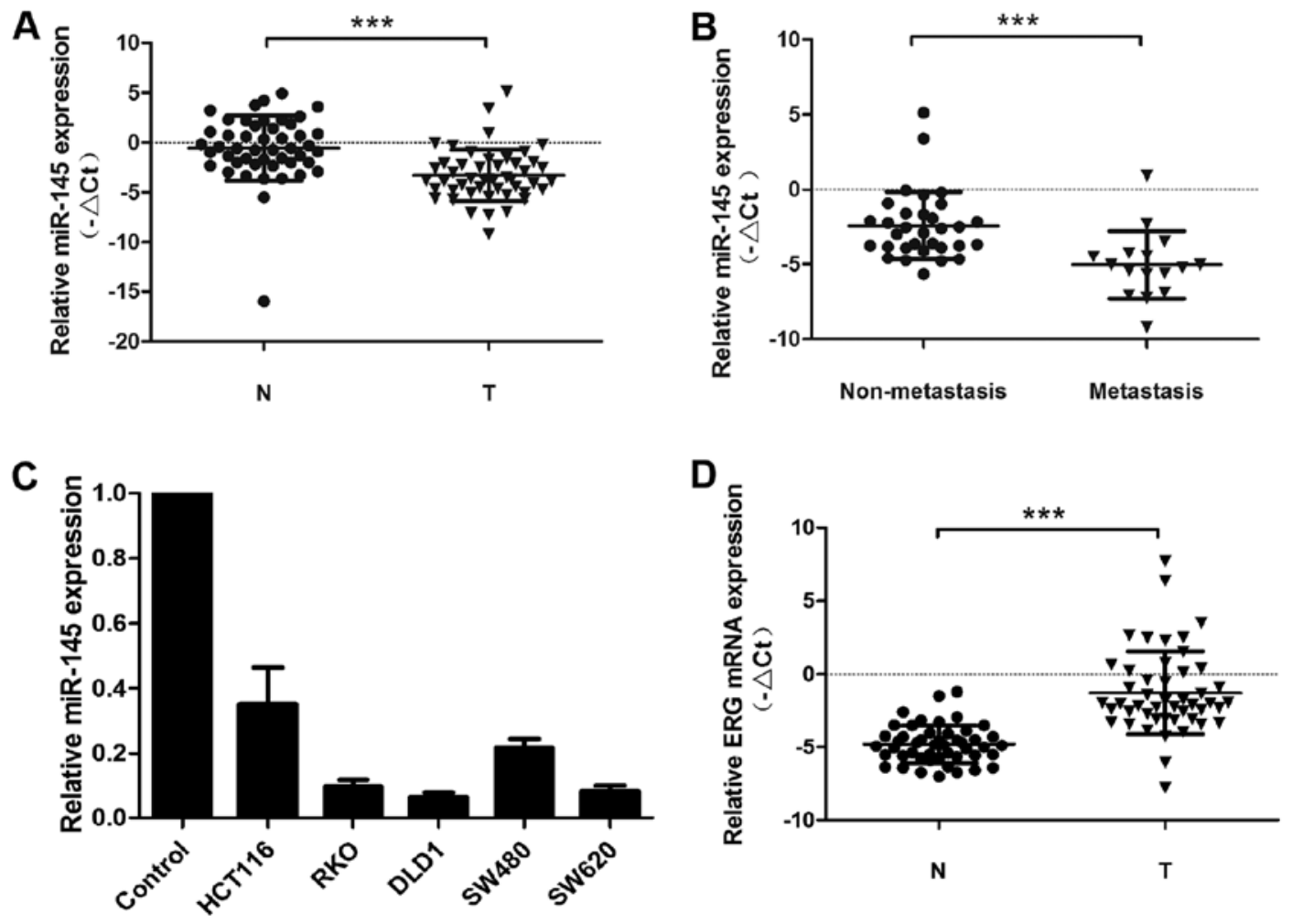

Figure 1. Downregulation of miR-145 expression in CRC cell lines and tissues. (A) qRT-PCR analysis of miR-145 expression in 48 pairs of CRC tissue and corresponding adjacent non-cancerous mucous tissue. $\Delta \mathrm{Ct}$ represents miR-145 expression normalized to U6. (B) miR-145 expression in primary CRC of patients with or without lymph node metastasis. (C) miR-145 expression in five human colorectal cancer cell lines compared to adjacent non-cancerous mucous tissue. (D) ERG expression in CRC tissue and adjacent non-cancerous colorectal mucosa. ${ }^{* * *} \mathrm{P}<0.001$. $\mathrm{N}$, adjacent non-cancer mucosa; $\mathrm{T}$, colorectal tumor tissue.

miR-145 binding site in the ERG 3'-UTR region was also constructed and denoted as psiCHECK2-MUT. The primers used for these constructs were as follows: psiCHECK2-WT forward, 5'-CGCGCGATCGCAGACCTGGCGGAGGCT TTTC-3' and reverse, 5'-ATAAGAATGCGGCCGCGGCTC TCCCTTGCACAAGTTC-3'; psiCHECK2-MUT forward, 5'-TCTTTGTTTGTCAAATGAAAATTTTTTCCAGTTTTG TCTGATATTTAAGAGAAACATT-3' and reverse, 5'-AATGTTTCTCTTAAATATCAGACAAAACTGGAA AAAATTTTCATTTGACAAACAAAGA-3’. For Dual-Luciferase assays, $50 \mathrm{nM}$ of miR-145 mimics or $100 \mathrm{nM}$ of miR-145 inhibitor were co-transfected with $0.5 \mu \mathrm{g}$ of psiCHECK2-WT or psiCHECK2-MUT using Lipofectamine 2000 (Invitrogen Life Technologies). The renilla luciferase signal and the firefly luciferase signal were consecutively detected according to the manufacturer's protocols for Dual-Luciferase Reporter assay system (E1910) (Promega). The firefly luciferase signal was used to normalize the renilla luciferase signal.

Scratch wound healing assay. For the scratch wound healing assay, cells $\left(1 \times 10^{6}\right)$ were seeded homogeneously on 6-well plates and cultured for $24 \mathrm{~h}$ to form a monolayer. Next, monolayers were scratched carefully with a sterile plastic $200 \mu \mathrm{l}$ pipette tip. The floating cell debris was washed with DMEM. At 0 and $24 \mathrm{~h}$ after scratch would formation, images were obtained using an inverted microscope (Nikon, Tokyo, Japan) at a magnification of 200x and measured by Image-Pro Plus software (Media Cybernetics, Inc., Rockville, MD, USA).
Invasion assay. For invasion assays, Falcon inserts were used with Falcon Companion Tissue Culture Plates (Corning, Inc., Corning, NY, USA) (24 wells). The Falcon Cell Culture inserts with $8-\mu \mathrm{m}$ pore membranes were coated with Matrigel (BD Biosciences, San Jose, CA, USA) and placed in a $37^{\circ} \mathrm{C}$ incubator for $3 \mathrm{~h}$ to solidify. Homogeneous single cell suspensions with serum-free medium were added to the upper chamber at a total of $5 \times 10^{5}$ cells per well. Medium containing $10 \%$ FBS was added to the lower chambers and served as a chemoattractant. After $24 \mathrm{~h}$, cells that remained on the upper surface of the membrane were carefully removed by a cotton swab, and cells which invaded the pores and adhered to the lower surface of the membranes were fixed with methanol and stained with hematoxylin. Stained cells were observed and counted (five random x100 magnification fields per well) under an inverted microscope. Each experiment was performed in triplicate.

Statistical analysis. Statistical analysis was performed using SPSS 18.0 (IBM SPSS, Chicago, IL, USA). Results of all experiments are presented as mean \pm SD. Student's t-test was used to compare miRNA expression in clinical tissue samples and CRC cell lines. Pearson's correlation coefficient was used to measure the correlation between miR-145 and ERG expression. $\mathrm{P}<0.05$ was considered statistically significant.

\section{Results}

miR-145 is decreased in colorectal cancer cell lines and clinical samples. In this study we examined miR-145 expres- 
Table I. miR-145 expression and clinicopathological parameters of CRC patients and their tumors.

\begin{tabular}{|c|c|c|c|c|}
\hline \multirow{2}{*}{$\begin{array}{l}\text { Clinicopathological } \\
\text { parameters }\end{array}$} & \multirow{2}{*}{$\begin{array}{l}\text { No. of patients } \\
\qquad(\mathrm{N}=48)\end{array}$} & \multicolumn{2}{|c|}{ miR-145 expression } & \multirow[b]{2}{*}{ P-value } \\
\hline & & Low & High & \\
\hline \multicolumn{5}{|l|}{ Age (years) } \\
\hline$\leq 65$ & 13 & 8 & 5 & \multirow[t]{2}{*}{0.0569} \\
\hline$>65$ & 35 & 17 & 18 & \\
\hline \multicolumn{5}{|l|}{ Gender } \\
\hline Female & 19 & 9 & 10 & \multirow[t]{2}{*}{0.6675} \\
\hline Male & 29 & 16 & 13 & \\
\hline \multicolumn{5}{|l|}{$\mathrm{LN}$ involvement } \\
\hline No & 32 & 9 & 23 & \multirow[t]{2}{*}{$<0.0001^{\mathrm{a}}$} \\
\hline Yes & 16 & 16 & 0 & \\
\hline \multicolumn{5}{|l|}{ Distant metastasis } \\
\hline No & 36 & 13 & 23 & \multirow[t]{2}{*}{$<0.0001^{\mathrm{a}}$} \\
\hline Yes & 12 & 12 & 0 & \\
\hline \multicolumn{5}{|l|}{ TNM stage } \\
\hline I, II & 29 & 6 & 23 & \multirow[t]{2}{*}{$<0.0001^{\mathrm{a}}$} \\
\hline III, IV & 19 & 19 & 0 & \\
\hline
\end{tabular}

${ }^{\text {aS }}$ tatistically significant. miR-145, microRNA-145; CRC, colorectal cancer; LN, lymph node.

sion in 48 pairs of CRC tissues and adjacent non-cancerous mucosa samples by qRT-PCR (Fig. 1A). In this sample set, miR-145 expression was decreased in most of the CRC samples compared to non-cancerous adjacent mucous tissue. Moreover, even greater miR-145 downregulation was detected in CRC tumors obtained from patients with lymph node metastasis compared to those without lymph node metastasis (Fig. 1B). miR-145 expression in colorectal cancer cell lines (RKO, DLD-1, HCT-116, SW620 and SW480) was also decreased compared to adjacent non-cancerous mucous tissue (Fig. 1C). In contrast to miR-145 expression, ERG expression levels were upregulated in most CRC tissues compared to adjacent normal mucosa (Fig. 1D). In addition, we examined the relationship between miR-145 expression and clinicopathological features of CRC patients, including gender, age, lymph node metastasis, distant metastasis and TNM stage (Table I). The mean value of relative miR-145 expression (-2.7566) was used as a cut-off value for the division of CRC patients into miR-145 low or high expression groups, accordingly. Statistical analysis revealed that low miR-145 expression correlated with poor outcome indicators including lymph node metastasis $(\mathrm{P}<0.0001)$, distant metastasis $(\mathrm{P}<0.0001)$ and TNM stage $(\mathrm{P}<0.0001)$.

miR-145 suppresses migration and invasion ability of human CRC cells. We examined miR-145 in human CRC cell lines using the wound healing and Transwell assays. Transient transfection of miR-145 mimic, miR-145 inhibitor and their corresponding control RNAs was performed in CRC cell lines RKO and SW480. The wound healing assay revealed that upregulation of miR-145 using the miR-145 mimic transfection suppressed the migration rate of CRC cells compared to control cells (Fig. 2A), while miR-145 knockdown in miR-145 inhibitor transfection accelerated the migration rate compared to the control cells (Fig. 3A). Matrigel Transwell assays showed similar results. miR-145 overexpression in RKO and SW480 significantly suppressed the invasion ability of the cells (Fig. 2B), while the miR-145 knockdown increased the number of invaded cells (Fig. 3B). Collectively these results indicate a possible tumor suppressive role of miR-145 in CRC migration and invasion.

ERG is targeted by miR-145 in CRC cells. As miRNA exerts its function by interacting with the 3'-UTR of its target genes, bioinformatic algorithms were applied for target prediction. ERG was predicted to be a potential target of miR-145 (Fig. 4A). Based on this prediction, western blot analysis was performed to investigate the effects of ectopic expression of miR-145 in CRC cells. In our study miR-145 upregulation suppressed ERG protein expression in human CRC cell lines, RKO and SW480, while miR-145 knockdown resulted in increased ERG protein expression (Fig. 4B). Results of Dual-Luciferase reporter assays in HEK-293T cells revealed that miR-145 mimic transfection reduced the luciferase activity, while mutation in the predicted targeting region abrogated this suppressive effect (Fig. 4C). These results indicate that the interaction between miR-145 and ERG depended on the miRNA recognition element in the 3'-UTR region. Furthermore, miR-145 and ERG protein expression in eight colorectal cancer tissue samples was examined both by qRT-PCR and western blot analysis, respectively, and an inverse correlation was observed between miR-145 and ERG expression (Fig. 4D).

Downregulation of ERG partially reverses the effect of miR-145 knockdown in CRC cells. To further investigate 
A

RKO

\section{SW480}
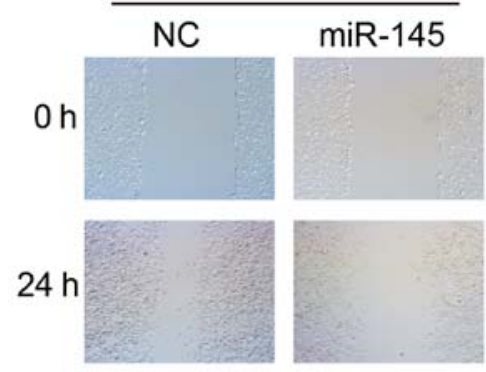

B
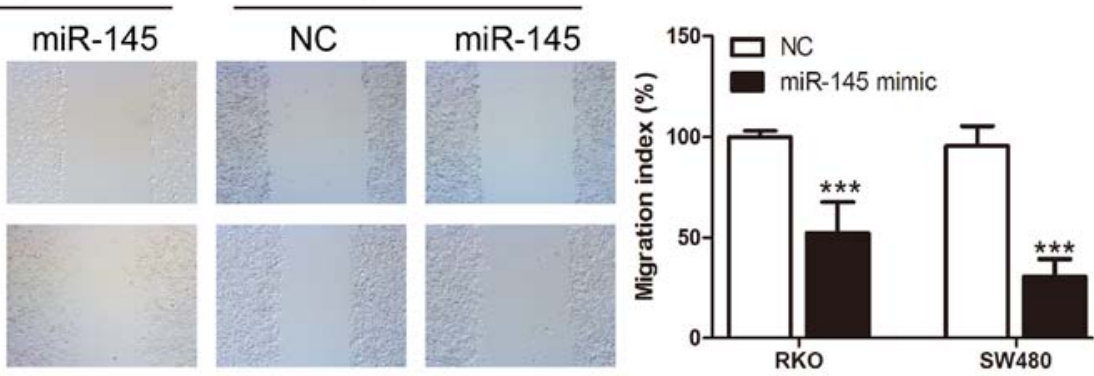

RKO

SW480
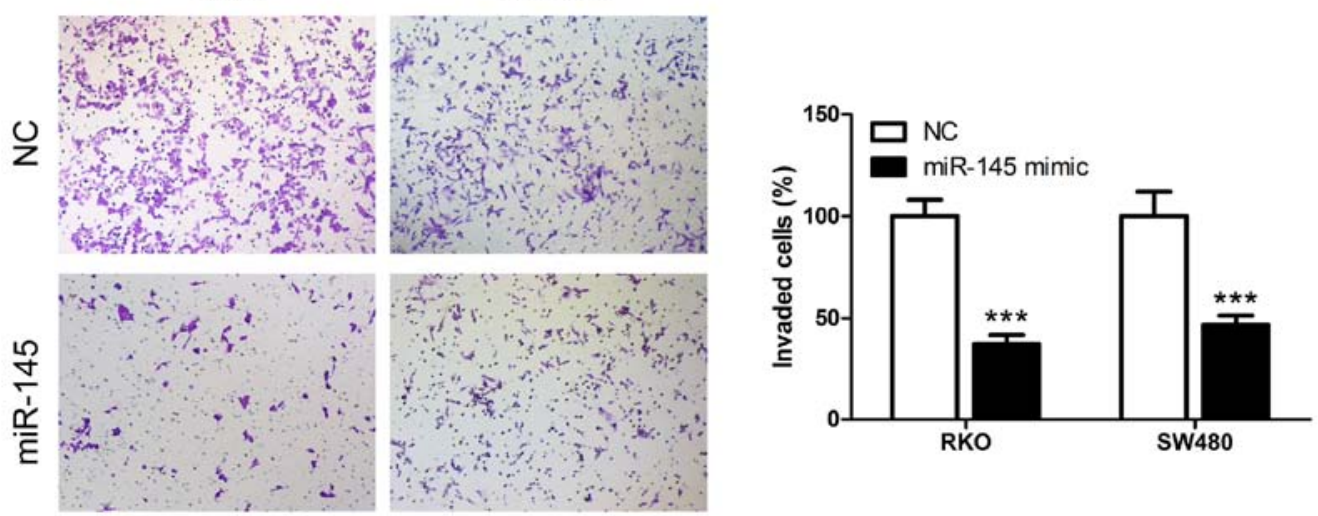

Figure 2. miR-145 suppressed migration and invasion of colorectal cancer cells. (A) Scratch wound healing assay revealed that miR-145 mimic transfection significantly reduced the horizontal migration ability of CRC cells (RKO and SW480) compared to the mimic control transfected cells. (B) Matrigel Transwell assay revealed that miR-145 mimic transfection significantly reduced the number of invaded cells compared to the mimic control transfected cells. ${ }^{* * * *} \mathrm{P}<0.001$.

A

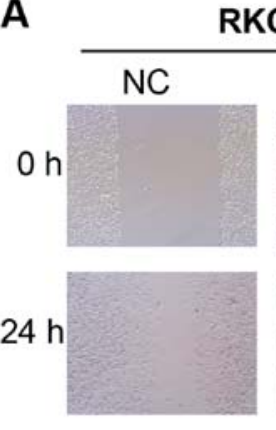

B
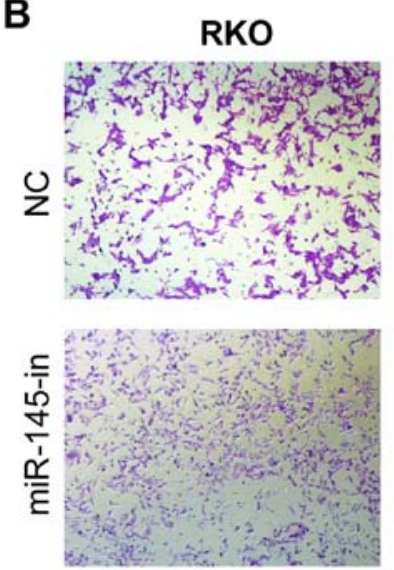

SW480
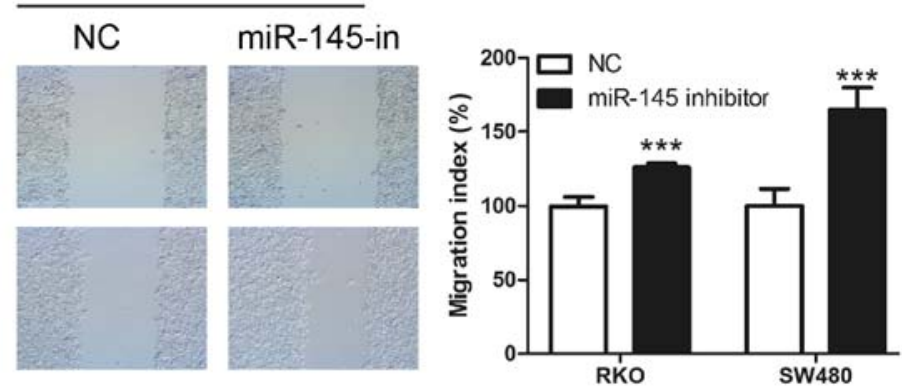

SW480
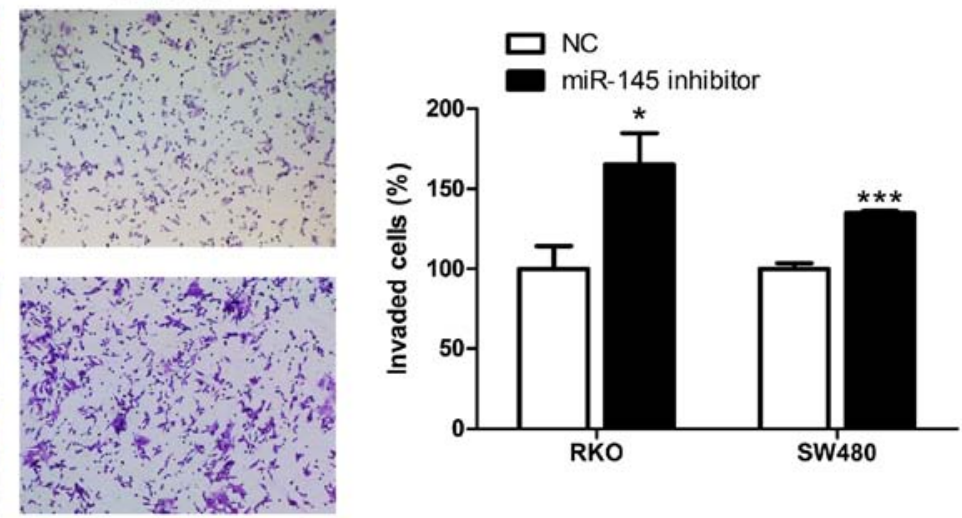

Figure 3. Downregulation of miR-145 promoted migration and invasion of colorectal cancer cells. (A) Scratch wound healing assay revealed that miR-145 inhibitor transfection significantly accelerated wound healing in CRC cells (RKO and SW480) compared to the inhibitor control transfected cells. (B) Matrigel Transwell assay revealed that miR-145 inhibitor transfection significantly increased the number of invaded cells compared to the inhibitor control transfected cells. ${ }^{*} \mathrm{P}<0.05,{ }^{* * * *} \mathrm{P}<0.001$. 
A
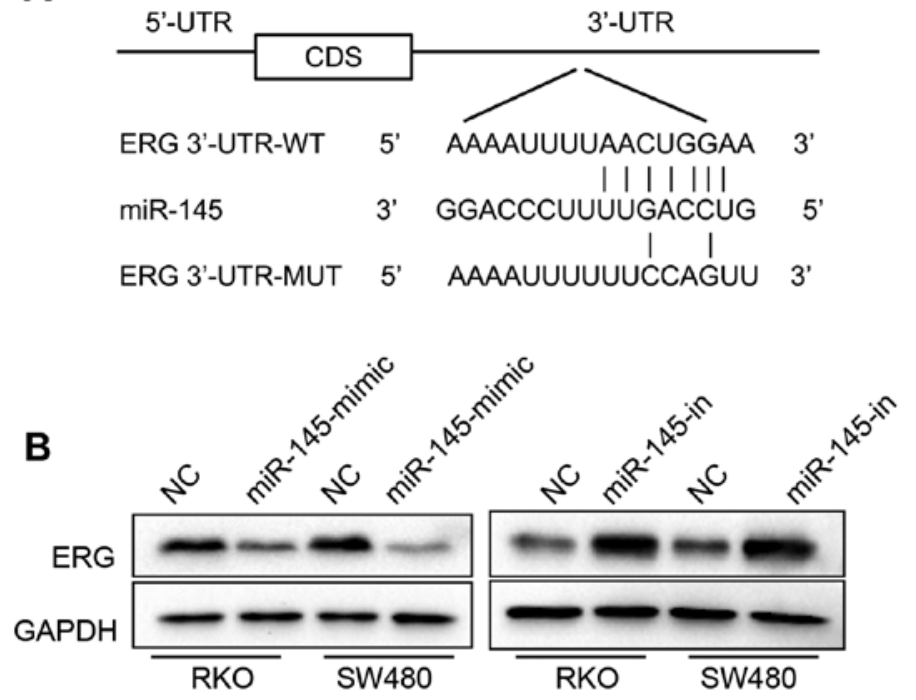

C

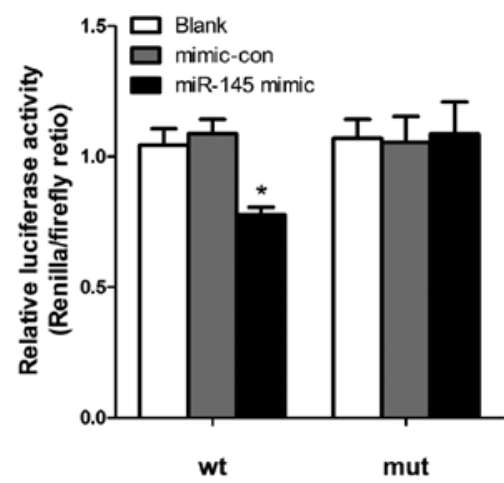

D
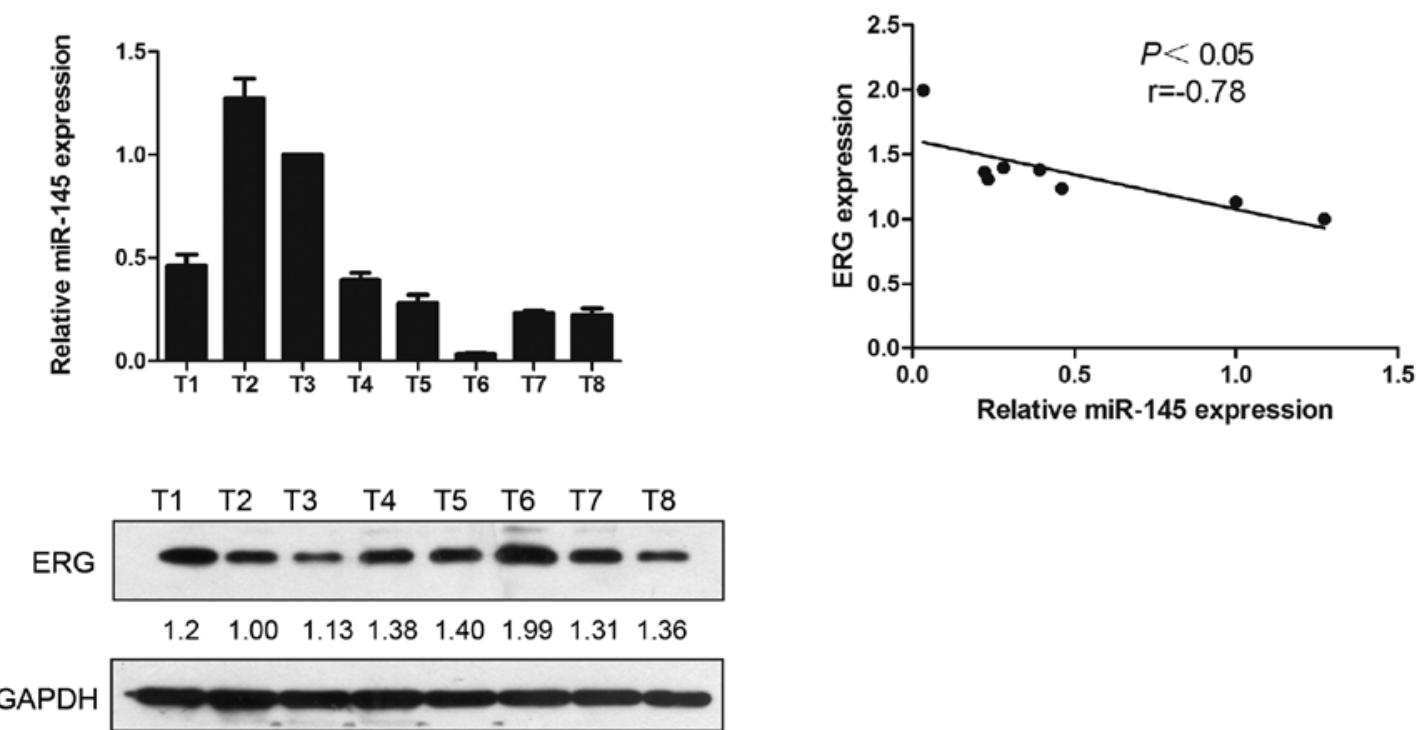

Figure 4. miR-145 downregulates ERG expression through prost-transcriptional regulation in CRC cells. (A) Schematic representation of the putative miR-145 target site in the ERG 3'-UTR. The original or mutant binding site of miR-145 in the 3'-UTR of ERG was inserted into the Dual-Luciferase plasmid. (B) Western blot analysis indicated that miR-145 mimic transfection suppressed ERG protein levels, while anti-miR-145 inhibitor transfection generated the opposite effect, compared to their negative controls, respectively. (C) Transfection of miR-145 mimics in HEK-293T cells resulted in decreased luciferase activity of the reporter plasmid containing the wild-type ERG 3'-UTR compared to cells transfected with mimic control. This effect was abrogated by the mutation of the complementary site in the $3^{\prime}$-UTR of ERG mRNA. " $\mathrm{P}<0.05$. (D) The relative expression of miR-145 represents $2^{-\Delta \Delta C t}$ normalized to sample T3. ERG expression is presented as the ration of ERG/GAPDH normalized to sample T2, in which ERG expression was the lowest. The ERG protein level in eight CRC tissues showed an inverse correlation with miR-145 $(\mathrm{P}<0.05, \mathrm{r}=-0.78)$.

whether miR-145 exerts its function in CRC cells through ERG regulation, siRNA targeting ERG was synthetized and transfected into CRC cell lines RKO and SW480. In our experiments, siRNA transfection downregulated the ERG protein level (Fig. 5A). Moreover, miR-145 inhibitor transfection increased ERG levels, while miR-145 inhibitor and ERG siRNA co-transfection partially recovered the ERG expression compared to cells transfected only with ERG siRNA (Fig. 5A). In the invasion experiments Matrigel Transwell assay revealed that ERG siRNA suppressed the invasiveness of RKO and SW480. In our study ERG siRNA transfection decreased the number of invaded cells compared to the negative control, while transfection with miR-145 inhibitors increased the number of invading cells. Co-transfection of miR-145 inhibitor and ERG siRNA recovered, to some degree, the invasive properties of RKO cells promoted by the miR-145 inhibitor (Fig. 5B).

\section{Discusion}

Over the past few decades, research efforts and improved understanding of the carcinogenesis and progression of colorectal cancer have contributed to its better diagnosis opening new avenues for targeted therapy. However, the efficacy of targeted therapy remains unsatisfactory due to our incomplete understanding of the tumor pathology and cell signaling pathways (20). 


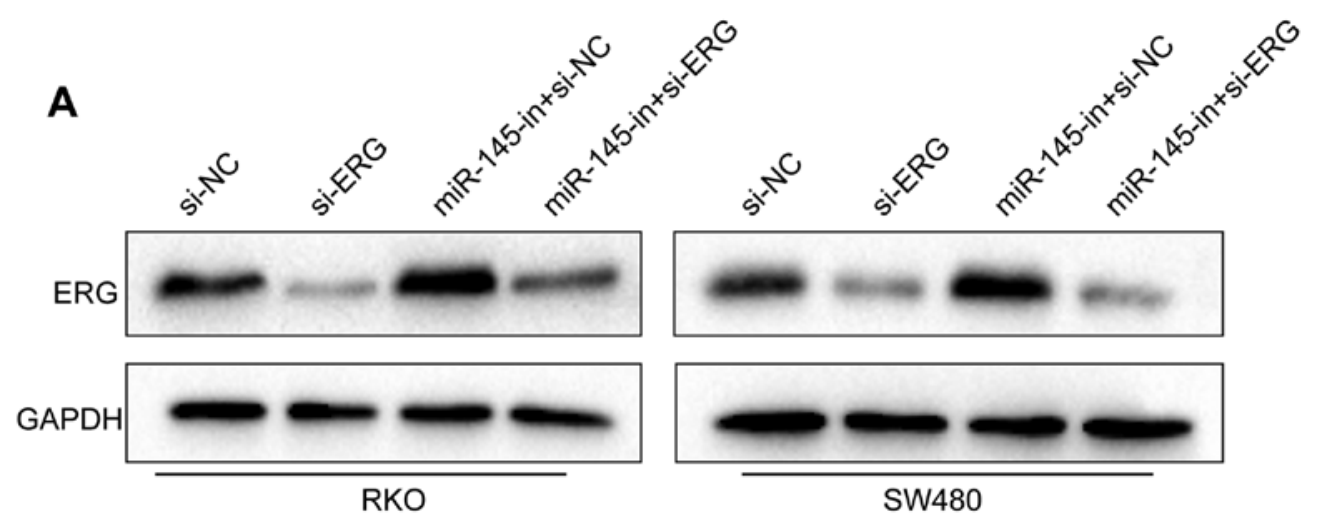

B

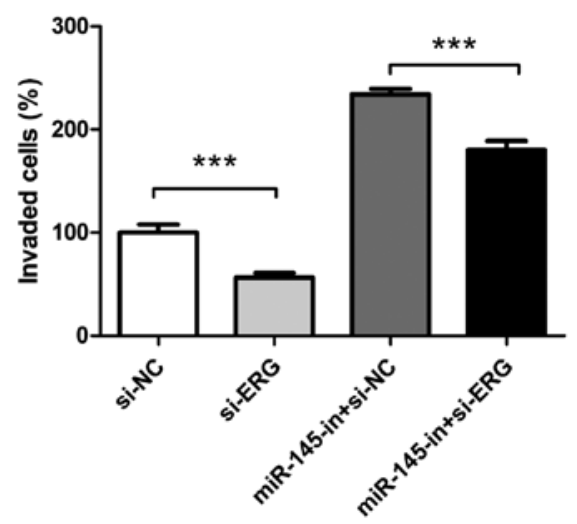

Figure 5. Inhibition of ERG expression by ERG siRNA partially reverses the effect of miR-145 knockdown in CRC cells. (A) Western blot analysis revealed that transfection of ERG siRNA into RKO cells resulted in decreased ERG expression compared to the cells transfected with scrambled siRNA. These effects of siRNA were attenuated by anti-miR-145 inhibitor transfection. (B) Transwell assays revealed that ERG siRNA transfection suppressed the invasiveness of RKO and SW480 cells. Co-transfection of ERG siRNA and anti-miR-145 inhibitors abrogated the suppressive effect caused by ERG knockdown. ${ }^{* * *} \mathrm{P}<0.001$.

Previous studies have shown that miR-145 is commonly downregulated in various types of cancer, including CRC, which indicated the possible role of miR-145 in carcinogenesis and tumor progression (21). With regards to its expression, miR-145 was found to be persistently decreased in adenoma and colorectal neoplasms (12). In addition, miR-145 downregulation was reported in ovarian cancer (22), cervical cancer (23), lung cancer (24), and gastric cancer (25). Previous functional studies have shown that miR-145 transfection represses CRC cell proliferation, invasion and metastasis by directly suppressing fascin-1 (26) and paxillin (27). In addition, miR-145 transfection negatively regulated IGF-1R expression and suppressed proliferation of Caco-2 cells (28). Furthermore, other recent studies reported that miR-145 could suppress RAD18 expression and consequently enhance DNA damage in CRC cells after 5-FU treatment, therefore implying that there is a drug-resistance reversal effect of miR-145 (29). Moreover, upregulation of miR-145 increased the sensitivity of drug-resistant Colo205 cells to vemurafenib both in vitro and in vivo (30). In a study by Pagliuca et al, resaturation of miR-145 and miR-143 in colon cancer cells showed that by targeting CD44, KLF5, KRAS and BRAF, miR-145 and miR-143 there was a resultant coordinated decrease in proliferation, migration and chemoresistance of colon cancer cells (31). Therefore, downregulation of miR-145 might play an important role in the tumorigenesis and progression of colorectal cancer and further studies on
miR-145 in cancer might bring new avenues for miR-145 targeted therapy.

Our previous study (32) examined the miRNA expression profiles of 31 pairs of CRC tissues and adjacent non-cancerous tissues and miR-145 was downregulated in most of the clinical samples. Thus, we further explored the role of miR-145 in colorectal tumorigenesis. Consistent with previous studies and our earlier findings (32), miR-145 expression was downregulated in both clinical CRC samples, as well as in cell lines, compared to normal colorectal tissue samples. Moreover, miR-145 expression in primary tumors taken from patients with lymph node metastasis was significantly lower than in tumors of patients without lymph node metastasis. This finding indicates a potential tumor-suppressive role of miR-145 in tumor metastasis. Moreover, miR-145 transfection in RKO and SW480 CRC cell lines significantly reduced cell migration and invasiveness. Contrary to these findings, miR-145 downregulation via the miR-145 inhibitor promoted migration and invasion of CRC cells compared to untreated cells.

In contrast to a majority of studies, a few studies have proposed that miR-145 is CRC oncogenic. For instance, Yuan et al reported that miR-145 promoted HCT- 8 colon cancer cell metastasis by stabilizing Hsp-27, a protein associated with metastasis (33). In another study by Arndt et al miR-145 promoted cell proliferation and anchorage-independent growth by downregulating E-cadherin in the colorectal 
cancer cell line SW620 (17). The fact that a specific miRNA can regulate various target genes may explain its dual and even contradictory influence in tumor initiation and progression. Indeed, diverse cellular contexts, tumor types and target gene sequence variations may explain why a specific miRNA might have diverse functions in tumor initiation and progression $(34,35)$. Based on the present results and our previous findings (36) we propose that miR-145 acts as a tumor suppressor during the progression of CRC.

ERG is one of the transcription factors belonging to the ETS family, which is one of the largest families of transcriptional regulators with diverse functions and activities (37). All members of the ETS family functionally regulate carcinogenesis-related processes such as cell proliferation, angiogenesis, apoptosis and metastasis $(38,39)$. Previous studies have demonstrated that ETS genes function as proto-oncogenes in colon cancer, and ETS-1 and -2 expression was associated with lymph node metastasis and advanced tumor grade in colon cancer (40). Ectopic expression of ERG was demonstrated in other tumors, such as acute myeloid leukemia and Ewing's sarcoma (41). Furthermore, invasive breast cancer mRNA expression datasets reflected a general ERG-driven pattern of malignancy (39). Emerging evidence suggest that ERG overexpression is involved in oncogenesis and progression of various cancer types; however, little is known about the regulation of ERG in CRC carcinogenesis. Interestingly, other members of the ETS family have been reported to be associated with tumor grade and metastasis status in colon cancer (42). Furthermore, aberrant expression of ERG and ETS-2 were associated with the development of cervical carcinoma (43). In addition, Scheble et al suggested that the TMPRSS2-ERG gene fusion, which can lead to overexpression of ERG, is specific for prostate cancer, and no such gene fusion was observed in CRC (44). Recent studies identified various miRNAs that can regulate ETS. For example, miR-196a and b act as ERG regulators in acute leukemia (45). miR-145 directly targets ERG in prostate cancer and suppresses proliferation of prostate cancer cells (46). Furthermore, miR-145 inhibits expression of ETS-1 in gastric cancer cells and exerts its suppressive effect towards invasion, metastasis and angiogenesis by regulating the genes downstream of ETS-1, such as matrix metalloproteinase-1 and -9 (47). miR-139 suppresses ETS-1 expression in CRC cells and inhibits cell proliferation and G1/S phase cell cycle transition (48). Thus, based on these findings we hypothesized that some regulatory mechanisms other than transcriptional regulation might contribute to the dysregulation of ERG observed in various cancer types including CRC. Our current results showed that ERG was upregulated in CRC cancer specimens compared to corresponding adjacent normal tissue. Based on the computational algorithm analysis, we speculated that miR-145 directly regulates ERG through interaction with the 3'-UTR of ERG mRNA. Our results confirmed our hypothesis since the downregulation of miR-145 increased ERG expression, while the restoration of miR-145 resulted in decreased ERG protein expression in CRC cells. Dual-Luciferase reporter assay suggested that miR-145-mediated suppression of ERG is dependent on the 3'-UTR of ERG mRNA. miR-145 expression inversely correlated with ERG protein levels, further confirming the negative regulation of ERG by miR-145. Moreover, ERG knockdown suppressed the invasion of CRC cells in vitro, demonstrating that ERG may serve as a proto-oncogene in CRC. Furthermore, co-transfection of ERG siRNA and miR-145 inhibitor showed that ERG downregulation partially, but not completely, reversed the tumor promoting effect caused by the miR-145 inhibitor. Taken together, these results indicate that ERG acts as a proto-oncogene in CRC cells.

Although it is not yet clear whether ERG is subjected to transcriptional regulation in CRC, this current study indicates that there is post-transcriptional regulation of ERG by miR-145 in CRC. Previous studies have shown that miR-145 inhibits colon cancer cell growth by targeting Friend leukemia virus integration-1 (FLI-1), another member of the ETS family of transcription factors (49). Furthermore, Ban et al reported feedback regulation between EWS-FLI-1 and Hsa-miR-145 in Ewing's sarcoma (50). Therefore, along with our results, it is reasonable to speculate that miR-145 may regulate different members of the ETS transcription factor family and consequently modulate diverse pathological processes in CRC. Further studies are needed to understand the roles of miR-145 and ERG in CRC metastasis, which might provide us with a novel therapy for the treatment of colorectal cancer.

In conclusion, our results show that miR-145 is persistently downregulated during CRC progression. In addition, miR-145 might suppress CRC cell proliferation and invasion through direct repression of the proto-oncogene ERG. Therefore, restoration of miR-145 and suppression of ERG might be utilized as a potential new therapeutic strategy in colorectal cancer in the future.

\section{Acknowledgements}

We appreciate the help generously offered by S.W., Z.L., R.C., X.W. and N.H. for the clinical sample collection. We also thank the facility and academic advice given by the Key Laboratory for Major Obstetric Diseases of Guangdong province and the Key Laboratory of Reproduction and Genetics of Guangdong Higher Education Institutes. This research was Supported by Science and Technology Planning Project of Guangdong Province, China (grant no. 2016B090918130); Medical Science and Technology Research Foundation of Guangdong Province (grant no. A2016500) and Youth Program of Guangzhou Medical University (grant no. 2014A10). We also greatly appreciate the funding support provided by Dr Anwei Chen.

\section{References}

1. Siegel R, Desantis C and Jemal A: Colorectal cancer statistics, 2014. CA Cancer J Clin 64: 104-117, 2014.

2. DeSantis CE, Lin CC, Mariotto AB, Siegel RL, Stein KD, Kramer JL, Alteri R, Robbins AS and Jemal A: Cancer treatment and survivorship statistics, 2014. CA Cancer J Clin 64: 252-271, 2014.

3. Loughran SJ, Kruse EA, Hacking DF, de Graaf CA, Hyland CD, Willson TA, Henley KJ, Ellis S, Voss AK, Metcalf D, et al: The transcription factor Erg is essential for definitive hematopoiesis and the function of adult hematopoietic stem cells. Nat Immunol 9: 810-819, 2008.

4. Birdsey GM, Dryden NH, Amsellem V, Gebhardt F, Sahnan K, Haskard DO, Dejana E, Mason JC and Randi AM: Transcription factor Erg regulates angiogenesis and endothelial apoptosis through VE-cadherin. Blood 111: 3498-3506, 2008. 
5. Iwamoto M, Tamamura Y, Koyama E, Komori T, Takeshita N, Williams JA, Nakamura T, Enomoto-Iwamoto $M$ and Pacifici M: Transcription factor ERG and joint and articular cartilage formation during mouse limb and spine skeletogenesis. Dev Biol 305: 40-51, 2007.

6. Gao X, Li LY, Zhou FJ, Xie KJ, Shao CK, Su ZL, Sun QP, Chen MK, Pang J, Zhou XF, et al: ERG rearrangement for predicting subsequent cancer diagnosis in high-grade prostatic intraepithelial neoplasia and lymph node metastasis. Clin Cancer Res 18: 4163-4172, 2012.

7. Marcucci G, Maharry K, Whitman SP, Vukosavljevic T, Paschka P, Langer C, Mrózek K, Baldus CD, Carroll AJ, Powell BL, et al; Cancer and Leukemia Group B Study: High expression levels of the ETS-related gene, ERG, predict adverse outcome and improve molecular risk-based classification of cytogenetically normal acute myeloid leukemia: A Cancer and Leukemia Group B Study. J Clin Oncol 25: 3337-3343, 2007.

8. Markert EK, Mizuno H, Vazquez A and Levine AJ: Molecular classification of prostate cancer using curated expression signatures. Proc Natl Acad Sci USA 108: 21276-21281, 2011.

9. Becker-Santos DD, Guo Y, Ghaffari M, Vickers ED, Lehman M, Altamirano-Dimas M, Oloumi A, Furukawa J, Sharma M, Wang Y, et al: Integrin-linked kinase as a target for ERG-mediated invasive properties in prostate cancer models. Carcinogenesis 33: 2558-2567, 2012.

10. O'Donnell KA, Wentzel EA, Zeller KI, Dang CV and Mendell JT: c-Myc-regulated microRNAs modulate E2F1 expression. Nature 435: 839-843, 2005.

11. Esquela-Kerscher A and Slack FJ: Oncomirs - microRNAs with a role in cancer. Nat Rev Cancer 6: 259-269, 2006.

12. Michael MZ, O' Connor SM, van Holst Pellekaan NG, Young GP and James RJ: Reduced accumulation of specific microRNAs in colorectal neoplasia. Mol Cancer Res 1: 882-891, 2003.

13. Iorio MV, Ferracin M, Liu CG, Veronese A, Spizzo R, Sabbioni S Magri E, Pedriali M, Fabbri M, Campiglio M, et al: MicroRNA gene expression deregulation in human breast cancer. Cancer Res 65: 7065-7070, 2005.

14. Watahiki A, Wang Y, Morris J, Dennis K, O'Dwyer HM, Gleave M, Gout PW and Wang Y: MicroRNAs associated with metastatic prostate cancer. PLoS One 6: e24950, 2011.

15. Zhang H, Pu J, Qi T, Qi M, Yang C, Li S, Huang K, Zheng L and Tong Q: MicroRNA-145 inhibits the growth, invasion, metastasis and angiogenesis of neuroblastoma cells through targeting hypoxia-inducible factor 2 alpha. Oncogene 33: 387-397, 2014.

16. Panza A, Votino C, Gentile A, Valvano MR, Colangelo T, Pancione M, Micale L, Merla G, Andriulli A, Sabatino L, et al: Peroxisome proliferator-activated receptor $\gamma$-mediated induction of microRNA-145 opposes tumor phenotype in colorectal cancer. Biochim Biophys Acta 1843: 1225-1236, 2014.

17. Arndt GM, Dossey L, Cullen LM, Lai A, Druker R, Eisbacher M, Zhang C, Tran N, Fan H, Retzlaff K, et al: Characterization of global microRNA expression reveals oncogenic potential of miR-145 in metastatic colorectal cancer. BMC Cancer 9: 374, 2009.

18. Benson AB III, Arnoletti JP, Bekaii-Saab T, Chan E, Chen YJ, Choti MA, Cooper HS, Dilawari RA, Engstrom PF, Enzinger PC, et al; National Comprehensive Cancer Network: Anal Carcinoma, Version 2.2012: Featured updates to the NCCN guidelines. J Natl Compr Canc Netw 10: 449-454, 2012.

19. Schindelin J, Rueden CT, Hiner MC and Eliceiri KW: The ImageJ ecosystem: An open platform for biomedical image analysis. Mol Reprod Dev 82: 518-529, 2015.

20. Sameer AS: Colorectal cancer: Molecular mutations and polymorphisms. Front Oncol 3: 114, 2013.

21. Luo X, Burwinkel B, Tao S and Brenner H: MicroRNA signatures: Novel biomarker for colorectal cancer? Cancer Epidemiol Biomarkers Prev 20: 1272-1286, 2011.

22. Iorio MV, Visone R, Di Leva G, Donati V, Petrocca F, Casalini P, Taccioli C, Volinia S, Liu CG, Alder H, et al: MicroRNA signatures in human ovarian cancer. Cancer Res 67: 8699-8707, 2007.

23. Wang X, Tang S, Le SY, Lu R, Rader JS, Meyers C and Zheng ZM: Aberrant expression of oncogenic and tumor-suppressive microRNAs in cervical cancer is required for cancer cell growth. PLoS One 3: e2557, 2008.

24. Liu X, Sempere LF, Galimberti F, Freemantle SJ, Black C, Dragnev KH, Ma Y, Fiering S, Memoli V, Li H, et al: Uncovering growth-suppressive MicroRNAs in lung cancer. Clin Cancer Res 15: 1177-1183, 2009.
25. Takagi T, Iio A, Nakagawa Y, Naoe T, Tanigawa N and Akao Y: Decreased expression of microRNA-143 and -145 in human gastric cancers. Oncology 77: 12-21, 2009.

26. Dong R, Liu X, Zhang Q, Jiang Z, Li Y, Wei Y, Li Y, Yang Q, Liu J, Wei JJ, et al: miR-145 inhibits tumor growth and metastasis by targeting metadherin in high-grade serous ovarian carcinoma. Oncotarget 5: 10816-10829, 2014

27. Qin J, Wang F, Jiang H, Xu J, Jiang Y and Wang Z: MicroRNA-145 suppresses cell migration and invasion by targeting paxillin in human colorectal cancer cells. Int J Clin Exp Pathol 8: 1328-1340, 2015.

28. Shi B, Sepp-Lorenzino L, Prisco M, Linsley P, deAngelis T and Baserga R: Micro RNA 145 targets the insulin receptor substrate-1 and inhibits the growth of colon cancer cells. J Biol Chem 282: 32582-32590, 2007.

29. Liu RL, Dong Y, Deng YZ, Wang WJ and Li WD: Tumor suppressor miR-145 reverses drug resistance by directly targeting DNA damage-related gene RAD18 in colorectal cancer. Tumour Biol 36: 5011-5019, 2015

30. Peng W, Hu J, Zhu XD, Liu X, Wang CC, Li WH and Chen ZY: Overexpression of miR-145 increases the sensitivity of vemurafenib in drug-resistant colo205 cell line. Tumour Biol 35: 2983-2988, 2014

31. Pagliuca A, Valvo C, Fabrizi E, di Martino S, Biffoni M, Runci D, Forte S, De Maria R and Ricci-Vitiani L: Analysis of the combined action of miR-143 and miR-145 on oncogenic pathways in colorectal cancer cells reveals a coordinate program of gene repression. Oncogene 32: 4806-4813, 2013.

32. Xu XH, Wu XB, Wu SB, Liu HB, Chen R and Li Y: Identification of miRNAs differentially expressed in clinical stages of human colorectal carcinoma-an investigation in Guangzhou, China. PLoS One 9: e94060, 2014.

33. Yuan W, Sui C, Liu Q, Tang W, An H and Ma J: Up-regulation of microRNA-145 associates with lymph node metastasis in colorectal cancer. PLoS One 9: e102017, 2014

34. Liu $H$ and Kohane IS: Tissue and process specific microRNA-mRNA co-expression in mammalian development and malignancy. PLoS One 4: e5436, 2009.

35. Chaudhuri K and Chatterjee R: MicroRNA detection and target prediction: Integration of computational and experimental approaches. DNA Cell Biol 26: 321-337, 2007.

36. Wu X, Xu X, Li S, Wu S, Chen R, Jiang Q, Liu H, Sun Y, Li Y and $\mathrm{Xu}$ Y: Identification and validation of potential biomarkers for the detection of dysregulated microRNA by qPCR in patients with colorectal adenocarcinoma. PLoS One 10: e0120024, 2015

37. Seth A and Watson DK: ETS transcription factors and their emerging roles in human cancer. Eur J Cancer 41: 2462-2478, 2005 .

38. Dejana E, Taddei A and Randi AM: Foxs and Ets in the transcriptional regulation of endothelial cell differentiation and angiogenesis. Biochim Biophys Acta 1775: 298-312, 2007.

39. Mochmann LH, Neumann M, von der Heide EK, Nowak V, Kühl AA, Ortiz-Tanchez J, Bock J, Hofmann WK and Baldus CD: ERG induces a mesenchymal-like state associated with chemoresistance in leukemia cells. Oncotarget 5: 351-362, 2014.

40. Ito Y, Takeda T, Okada M and Matsuura N: Expression of ets-1 and ets-2 in colonic neoplasms. Anticancer Res 22: 1581-1584, 2002.

41. Salek-Ardakani S, Smooha G, de Boer J, Sebire NJ, Morrow M, Rainis L, Lee S, Williams O, Izraeli S and Brady HJ: ERG is a megakaryocytic oncogene. Cancer Res 69: 4665-4673, 2009.

42. Peng C, Gao H, Niu Z, Wang B, Tan Z, Niu W, Liu E, Wang J, Sun J, Shahbaz M, et al: Integrin $\alpha v \beta 6$ and transcriptional factor Ets-1 act as prognostic indicators in colorectal cancer. Cell Biosci 4: 53, 2014.

43. Simpson S, Woodworth CD and DiPaolo JA: Altered expression of Erg and Ets-2 transcription factors is associated with genetic changes at 21q22.2-22.3 in immortal and cervical carcinoma cell lines. Oncogene 14: 2149-2157, 1997.

44. Scheble VJ, Braun M, Beroukhim R, Mermel CH, Ruiz C, Wilbertz T, Stiedl AC, Petersen K, Reischl M, Kuefer R, et al: ERG rearrangement is specific to prostate cancer and does not occur in any other common tumor. Mod Pathol 23: 1061-1067, 2010.

45. Coskun E, von der Heide EK, Schlee C, Kühnl A, Gökbuget N, Hoelzer D, Hofmann WK, Thiel E and Baldus CD: The role of microRNA-196a and microRNA-196b as ERG regulators in acute myeloid leukemia and acute T-lymphoblastic leukemia. Leuk Res 35: 208-213, 2011. 
46. Hart M, Wach S, Nolte E, Szczyrba J, Menon R, Taubert H, Hartmann A, Stoehr R, Wieland W, Grässer FA, et al: The proto-oncogene ERG is a target of microRNA miR-145 in prostate cancer. FEBS J 280: 2105-2116, 2013.

47. Zheng L, Pu J, Qi T, Qi M, Li D, Xiang X, Huang K and Tong Q: miRNA-145 targets v-ets erythroblastosis virus E26 oncogene homolog 1 to suppress the invasion, metastasis, and angiogenesis of gastric cancer cells. Mol Cancer Res 11: 182-193, 2013

48. Gu J, Chen Y, Huang H, Yin L, Xie Z and Zhang MQ: Gene module based regulator inference identifying miR-139 as a tumor suppressor in colorectal cancer. Mol Biosyst 10: 3249-3254, 2014.
49. Zhang J, Guo H, Zhang H, Wang H, Qian G, Fan X, Hoffman AR, $\mathrm{Hu} J \mathrm{~F}$ and Ge S: Putative tumor suppressor miR-145 inhibits colon cancer cell growth by targeting oncogene Friend leukemia virus integration 1 gene. Cancer 117: 86-95, 2011.

50. Ban J, Jug G, Mestdagh P, Schwentner R, Kauer M, Aryee DN, Schaefer KL, Nakatani F, Scotlandi K, Reiter M, et al: Hsa-mir-145 is the top EWS-FLI1-repressed microRNA involved in a positive feedback loop in Ewing's sarcoma. Oncogene 30: 2173-2180, 2011. 